\title{
Choice Principles in Intuitionistic Set Theory
}

\author{
John L. Bell
}

In intuitionistic set theory, the law of excluded middle is known to be derivable from the standard version of the axiom of choice that every family of nonempty sets has a choice function. In this paper it is shown that each of a number of intuitionistically invalid logical principles, including the law of excluded middle, is, in intuitionistic set theory, equivalent to a suitably weakened version of the axiom of choice. Thus these logical principles may be viewed as choice principles.

We work in intuitionistic Zermelo-Fraenkel set theory IST (for a presentation, see [ 3 ], where it is called $\mathbf{Z F}^{\prime} \mathbf{r}$ ). Let us begin by fixing some notation. For each set $A$ we write $\mathrm{P} A$ for the power set of $A$, and $\mathrm{Q} X$ for the set of inhabited subsets of $A$, that is, of subsets $X$ of $A$ for which $\exists x(x \in A)$. The set of functions from $A$ to $B$ is denoted by $B^{A}$; the class of functions with domain $A$ is denoted by Fun $(A)$. The empty set is denoted by $0,\{0\}$ by 1 , and $\{0,1\}$ by 2 .

We tabulate the following logical schemes:

SLEM $\quad \alpha \vee \neg \alpha \quad(\alpha$ any sentence)

Lin $\quad(\alpha \rightarrow \beta) \vee(\beta \rightarrow \alpha) \quad(\alpha, \beta$ any sentences $)$

Stone $\quad \neg \alpha \vee \neg \neg \alpha \quad$ ( $\alpha$ any sentence)

Ex $\quad \exists x[\exists x \alpha(x) \rightarrow \alpha(x)] \quad(\alpha(x)$ any formula with at most $x$ free $)$

Un $\quad \exists x[\alpha(x) \rightarrow \forall x \alpha(x)] \quad(\alpha(x)$ any formula with at most $x$ free $)$

Dis $^{1} \quad \forall x[\alpha \vee \beta(x)] \rightarrow \alpha \vee \forall x \beta(x) \quad(\alpha$ any sentence, $\beta(x)$ any formula with at most $x$ free)

Over intuitionistic logic, Lin, Stone and Ex are consequences of SLEM; and Un implies Dis. All of these schemes follow, of course, from the full law of excluded middle, that is SLEM for arbitrary formulas.

We formulate the following choice principles-here $X$ is an arbitrary set and $\varphi(x, y)$ an arbitrary formula of the language of IST with at most the free variables $x, y$ :

\footnotetext{
${ }^{1}$ Dis is equivalent, over intuitionistic predicate logic, to what is called in [4] the higher dual distributive lawHDDL $\forall x[\alpha(x) \vee \beta(x)] \rightarrow \exists x \alpha(x) \vee \forall x \beta(x)$.
} 
$\begin{array}{cl}\mathbf{A C}_{X} & \forall x \in X \exists y \varphi(x, y) \rightarrow \exists f \in \operatorname{Fun}(X) \forall x \in X \varphi(x, f x) \\ \text { AC }_{X}^{*} & \exists f \in \operatorname{Fun}(X)[\forall x \in X \exists y \varphi(x, y) \rightarrow \forall x \in X \varphi(x, f x)] \\ \text { DAC }_{X} & \forall f \in \operatorname{Fun}(X) \exists x \in X \varphi(x, f x) \rightarrow \exists x \in X \quad \forall y \varphi(x, y) \\ \text { DAC }_{X}^{*} & \exists f \in \operatorname{Fun}(X)[\exists x \in X \varphi(x, f x) \rightarrow \exists x \in X \quad \forall y \varphi(x, y)]\end{array}$

The first two of these are forms of the axiom of choice for $X$; while classically equivalent, in IST $\mathbf{A C}{ }^{*}$ implies $\mathbf{A C} \mathbf{C}_{X}$, but not conversely. The principles $\mathbf{D A C} \mathbf{C}_{X}$ and $\mathbf{D A} \mathbf{C}_{X}^{*}$ are $d u a l$ forms of the axiom of choice for $X$ : classically they are both equivalent to $\mathbf{A C}_{X}$ and $\mathbf{A C}_{X}^{*}$, but in IST DAC ${ }_{X}^{*}$ implies DAC $\mathbf{C}_{X}$, and not conversely.

We also formulate what we shall call the weak extensional selection principle, in which $\alpha(x)$ and $\beta(x)$ are any formulas with at most the variable $x$ free:

WESP $\exists x \in 2 \alpha(x) \wedge \exists x \in 2 \beta(x) \rightarrow \exists x \in 2 \exists y \in 2[\alpha(x) \wedge \beta(y) \wedge[\forall x \in 2[\alpha(x) \leftrightarrow \beta(x)] \rightarrow x=y]]$.

This principle asserts that, for any pair of instantiated properties of members of 2 , instances may be assigned to the properties in a manner that depends just on their extensions. WESP is a straightforward consequence of $\mathbf{A C}_{Q_{2}}$. For taking $\varphi(u, y)$ to be $y \in u$ in $\mathbf{A C}_{Q 2}$ yields the existence of a function $f$ with domain $Q 2$ such that $f u \in u$ for every $u \in Q 2$. Given formulas $\alpha(x), \beta(x)$, and assuming the antecedent of WESP, the sets $U=\{x \in 2: \alpha(x)\}$ and $V=\{x \in 2: \beta(x)\}$ are members of $Q 2$, so that $a=f U \in U$, and $b=f V \in V$, whence $\alpha(a)$ and $\beta(b)$. Also, if $\forall x \in 2[\alpha(x) \leftrightarrow \beta(x)]$, then $U=V$, whence $a=b$; it follows then that the consequent of WESP holds.

We are going to show that each of the logical principles tabulated above is equivalent (over IST) to a choice principle. Starting at the top of the list, we have first:

- WESP and SLEM are equivalent over IST.

Proof. Assume WESP. Let $\sigma$ be any sentence and define

$$
\alpha(x) \equiv x=0 \vee \sigma \quad \beta(x) \equiv x=1 \vee \sigma .
$$

With these instances of $\alpha$ and $\beta$ the antecedent of WESP is clearly satisfied, so that there exist members $a, b$ of 2 for which (1) $\alpha(a) \wedge \beta(b)$ and (2) $\forall x[[\forall x \in 2[\alpha(x) \leftrightarrow \beta(x)] \rightarrow$ $a=b$. It follows from (1) that $\sigma \vee(a=0 \wedge b=1)$, whence (3) $\sigma \vee a \neq b$. And since clearly $\sigma \rightarrow \forall x \in 2[\alpha(x) \leftrightarrow \beta(x)]$ we deduce from (2) that $\sigma \rightarrow a=b$, whence $a \neq b \rightarrow \neg \sigma$. Putting this last together with (3) yields $\sigma \vee \neg \sigma$, and SLEM follows. 
For the converse, we argue informally. Suppose that SLEM holds. Assuming the antecedent of WESP, choose $a \in 2$ for which $\alpha(a)$. Now (using SLEM) define an element $b \in 2$ as follows. If $\forall x \in 2[\alpha(x) \leftrightarrow \beta(x)]$ holds, let $b=a$; if not, choose $b$ so that $\beta(b)$. It is now easy to see that $a$ and $b$ satisfy $\alpha(a) \wedge \beta(b) \wedge[\forall x \in 2[\alpha(x) \leftrightarrow \beta(x)] \rightarrow a=b]$. WESP follows.

Remark. The argument for WESP $\rightarrow$ SLEM is another "stripped down" version of Diaconescu's theorem that, in a topos, the axiom of choice implies the law of excluded middle. The result may be compared with that of [2] to the effect that the presence of extensional $\varepsilon$-terms renders intuitionistic logic classical.

Next, we observe that, while $\mathbf{A C} \mathbf{C}_{1}$ is (trivially) provable in IST, by contrast

- $\quad \mathbf{A C}_{1}^{*}$ and $\mathbf{E x}$ are equivalent over IST.

Proof. Assuming $\mathbf{A C}_{1}^{*}$, take $\varphi(x, y) \equiv \alpha(y)$ in its antecedent. This yields an $f \in \operatorname{Fun}(1)$ for which $\forall y \alpha(y) \rightarrow \alpha(f 0)$, giving $\exists y[\exists y \alpha(y) \rightarrow \alpha(y)]$, i.e., Ex.

Conversely, define $\alpha(y) \equiv \varphi(0, y)$. Then, assuming Ex, there is $b$ for which $\exists y \alpha(y) \rightarrow$ $\alpha(b)$, whence $\forall x \in 1 \exists y \varphi(x, y) \rightarrow \forall x \in 1 \varphi(x, b)$. Defining $f \in \operatorname{Fun}(1)$ by $f=\{\langle 0, b\rangle\}$ gives $\forall x \in 1 \exists y \varphi(x, y) \rightarrow \forall x \in 1 \varphi(x, f x)$, and $\mathbf{A C}_{1}^{*}$ follows.

Further, while $\mathbf{D A C} \mathbf{C}_{1}$ is easily seen to be provable in IST, we have

- $\quad \mathbf{D A C}_{1}^{*}$ and $\mathbf{U n}$ are equivalent over IST.

Proof. Given $\alpha$, Define $\varphi(x, y) \equiv \alpha(y)$. Then, for $f \in \operatorname{Fun}(1), \quad \exists x \in 1 \varphi(x, f x) \leftrightarrow \alpha(f 0)$ and $\exists x \in 1 \forall y \varphi(x, y) \leftrightarrow \forall y \alpha(y) . \mathbf{D A C}_{1}^{*}$ then gives

$$
\exists f \in \operatorname{Fun}(1)[\alpha(f 0) \rightarrow \forall y \alpha(y)]
$$

from which Un follows easily.

Conversely, given $\varphi$, define $\alpha(y) \equiv \varphi(0, y)$. Then from Un we infer that there exists $b$ for which $\alpha(b) \rightarrow \forall y \alpha(y)$, i.e. $\varphi(0, b) \rightarrow \forall y \varphi(0, y)$. Defining $f \in \operatorname{Fun}(1)$ by $f=\{\langle 0, b\rangle\}$ then gives $\varphi(0, f 0) \rightarrow \exists x \in 1 \forall y \varphi(x, y)$, whence $\exists x \in 1 \varphi(x, f x) \rightarrow \exists x \in 1 \forall y \varphi(x, y)$, and Un follows.

Next, while $\mathbf{A C _ { 2 }}$ is easily proved in IST, by contrast we have 
- $\quad \mathbf{D A C}_{2}$ and Dis are equivalent over IST.

Proof. The antecedent of $\mathbf{D A} \mathbf{C}_{2}$ is equivalent to the assertion

$$
\forall f \in \operatorname{Fun}(2)[\varphi(0, f 0) \vee \varphi(1, f 1)],
$$

which, in view of the natural correlation between members of Fun (2) and ordered pairs, is equivalent to the assertion

$$
\forall y \forall y\left\{\varphi(0, y) \vee \varphi\left(1, y^{\prime}\right)\right]
$$

The consequent of $\mathbf{D A C} \mathbf{C}_{2}$ is equivalent to the assertion

$$
\forall y \in Y \varphi(0, y) \vee \forall y^{\prime} \in Y \varphi\left(1, y^{\prime}\right)
$$

So $\mathbf{D A C} \mathbf{C}_{2}$ itself is equivalent to

$$
\forall y \forall y\left\{\varphi(0, y) \vee \varphi\left(1, y^{\prime}\right)\right] \rightarrow \forall y \varphi(0, y) \vee \forall y^{\prime} \varphi\left(1, y^{\prime}\right) .
$$

But this is obviously equivalent to the scheme

$$
\forall y \forall y\left[\alpha(y) \vee \beta\left(y^{\prime}\right)\right] \rightarrow \forall y \alpha(y) \vee \forall y^{\prime} \beta\left(y^{\prime}\right)
$$

where $y$ does not occur free in $\beta$, nor $y^{\prime}$ in $\alpha$. And this last is easily seen to be equivalent to Dis.

Now consider $\mathbf{D A C}{ }_{2}^{*}$. This is quickly seen to be equivalent to the assertion

$$
\exists z \exists z^{\prime}\left[\varphi(0, z) \vee \varphi\left(1, z^{\prime}\right) \rightarrow \forall y \varphi(0, y) \vee \forall y^{\prime} \varphi\left(1, y^{\prime}\right),\right.
$$

i.e. to the assertion, for arbitrary $\alpha(x), \beta(x)$, that

$$
\exists z \exists z^{\prime}\left[\alpha(z) \vee \beta\left(z^{\prime}\right) \rightarrow \forall y \alpha(y) \vee \forall y^{\prime} \beta\left(y^{\prime}\right)\right]
$$

This is in turn equivalent to the assertion, for any sentence $\alpha$,

$$
\exists y[\alpha \vee \beta(y) \rightarrow \alpha \vee \forall y \beta(y)]
$$

Now (*) obviously entails Un. Conversely, given Un, there is $b$ for which $\beta(b) \rightarrow \forall y \beta(y)$. Hence $\alpha \vee \beta(b) \rightarrow \alpha \vee \forall y \beta(y)$, whence $\left(^{*}\right)$. So we have shown that

- Over IST, $\mathbf{D A C} \mathbf{C}_{2}^{*}$ is equivalent to Un, and hence also to $\mathbf{D A C} \mathbf{C}_{1}^{*}$. In order to provide choice schemes equivalent to Lin and Stone we introduce 
$\operatorname{ac}_{X}^{*} \quad \exists f \in 2^{X}[\forall x \in X \exists y \in 2 \varphi(x, y) \rightarrow \forall x \in X \varphi(x, f x)]$

wac $_{X}^{*} \quad \exists f \in 2^{X}[\forall x \in X \exists y \in 2 \varphi(x, y) \rightarrow \forall x \in X \varphi(x, f x)]$ provided $\vdash$ IsT $\forall x[\varphi(x, 0) \rightarrow \neg \varphi(\mathrm{x}, 1)]$

Clearly $\mathbf{a c}{ }_{X}^{*}$ is equivalent to

$$
\exists f \in 2^{X}[\forall x \in X[\varphi(x, 0) \vee \varphi(x, 1)] \rightarrow \forall x \in X \varphi(x, f x)]
$$

and similarly for $\mathbf{w a c}_{X}^{*}$.

Then

- Over IST, $\mathbf{a c}{ }_{1}^{*}$ and $\mathbf{w a c}{ }_{1}^{*}$ are equivalent, respectively, to Lin and Stone.

Proof. Let $\alpha$ and $\beta$ be sentences, and define $\varphi(x, y) \equiv x=0 \wedge[(y=0 \wedge \alpha) \vee(y=1 \wedge \beta)]$. Then $\alpha \leftrightarrow \varphi(0,0)$ and $\beta \leftrightarrow \varphi(0,1)$, and so $\forall x \in 1[\varphi(x, 0) \vee \varphi(x, 1)] \leftrightarrow \varphi(0,0) \vee \varphi(0,1) \leftrightarrow \alpha \vee \beta$. Therefore

$$
\begin{aligned}
\exists f \in 2^{1}[\forall x \in 1[\varphi(x, 0) \vee \varphi(x, 1)] \rightarrow \forall x \in 1 \varphi(x, f x)] & \leftrightarrow \exists f \in 2^{1}[\alpha \vee \beta \rightarrow \varphi(0, f 0)] \\
& \leftrightarrow[\alpha \vee \beta \rightarrow \varphi(0,0)] \vee[\alpha \vee \beta \rightarrow \varphi(0,1)] \\
& \leftrightarrow[\alpha \vee \beta \rightarrow \alpha] \vee[\alpha \vee \beta \rightarrow \beta] \\
& \leftrightarrow \beta \rightarrow \alpha \vee \alpha \rightarrow \beta .
\end{aligned}
$$

This yields $\mathbf{a c}_{1}^{*} \rightarrow$ Lin. For the converse, define $\alpha \equiv \varphi(0,0)$ and $\beta \equiv \varphi(0,1)$ and reverse the argument.

To establish the second stated equivalence, notice that, when $\varphi(x, y)$ is defined as above, but with $\beta$ replaced by $\neg \alpha$, it satisfies the provisions imposed in $\mathbf{w a c}_{1}^{*}$. As above, that principle gives $(\neg \alpha \rightarrow \alpha) \vee(\alpha \rightarrow \neg \alpha)$, that is, $\neg \alpha \vee \neg \neg \alpha$. So Stone follows from $\mathbf{w a c}_{1}^{*}$. Conversely, suppose that $\varphi$ meets the condition imposed in $\mathbf{w a c}_{1}^{*}$ Then from $\varphi(0,0) \rightarrow \neg \varphi(0,1)$ we deduce $\neg \neg \varphi(0,0) \rightarrow \neg \varphi(0,1)$; now, assuming Stone, we have $\neg \varphi(0,0) \vee \neg \neg \varphi(0,0)$, whence $\neg \varphi(0,0) \vee \neg \varphi(0,1)$. Since $\neg \varphi(0,0) \rightarrow[\varphi(0,0) \rightarrow \varphi(0,1)]$ and $\neg \varphi(0,1) \rightarrow[\varphi(0,1) \rightarrow \varphi(0,0)]$ we deduce $[\varphi(0,0) \rightarrow \varphi(0,1)] \vee[\varphi(0,1) \rightarrow \varphi(0,0)]$. From the argument above it now follows that $\exists f \in 2^{1}[\forall x \in 1[\varphi(x, 0) \vee \varphi(x, 1)] \rightarrow \forall x \in 1 \quad \varphi(x, f x)]$. Accordingly $\mathbf{w a c}_{1}^{*}$ is a consequence of Stone.

In conclusion, we show how certain of the principles we have introduced can be derived in the presence of term-forming operators.

The $\varepsilon^{-}$and $\tau$-operators are term-forming operators yielding, for formulas $\alpha(x)$, terms $\varepsilon_{x} \alpha$ and $\tau_{x} \alpha$ in which the variable $x$ is no longer free; they are introduced in conjunction with the axioms-the $\varepsilon$ - and $\tau$-schemes: 


$$
\exists x \alpha(x) \rightarrow \alpha\left(\varepsilon_{x} \alpha\right) \quad \alpha\left(\tau_{x} \alpha\right) \rightarrow \forall x \alpha(x)
$$

It is an easy matter to derive $\mathbf{U n}$ from the $\tau$-scheme when $\tau$ is merely allowed to act on formulas with at most one free variable. When $\tau$ 's action is extended to formulas with two free variables, the $\tau$-scheme applied in IST yields the full dual axiom of choice $\forall X \mathbf{D A C}_{X}^{*}$. For under these conditions we have, for any formula $\varphi(x, y)$,

$$
\forall x \in X\left[\varphi\left(x, \tau_{y} \varphi(x, y)\right) \rightarrow \forall y \varphi(x, y)\right]
$$

Let $t \in \operatorname{Fun}(X)$ be the map $x \mapsto \tau_{y} \varphi(x, y)$. Assuming that $\forall f \in Y^{X} \exists x \in X \varphi(x, f x)$, let $a \in X$ satisfy $\varphi(a, t a)$. We deduce from $\left({ }^{*}\right)$ that $\forall y \in Y \varphi(a, y)$, whence $\exists x \in X \forall y \in Y \varphi(x, y)$. The dual axiom of choice follows.

In the case of the $\varepsilon$-operator, the number of free variables in the formulas on which the operator is allowed to act is an even more sensitive matter. If $\varepsilon$ is allowed to act only on formulas with at most one free variable (so yielding only closed terms), the corresponding $\varepsilon$-scheme applied in IST is easily seen to yield both $\mathbf{E x}$ and $\mathbf{a c} \mathbf{c}_{1}^{*}$, and so also Lin. But it is (in essence) shown in [1] that, if only closed $\varepsilon$-terms are admitted, SLEM is not derivable, and so therefore neither is WESP. The situation changes dramatically when $\varepsilon$ is permitted to operate on formulas with two free variables. For then from the corresponding $\varepsilon$-scheme it is easy to derive $\mathbf{A} \mathbf{C}_{X}$ for all sets $X$, and in particular $\mathbf{A} \mathbf{C}_{Q 2}$, and hence also SLEM.

I have found three ways of strengthening, or modifying, the single-variable $\varepsilon$ scheme so as to enable it to yield SLEM. The first, presented originally in [2], is to add to the $\varepsilon$-scheme Ackermann's Extensionality Principle, viz.

$$
\forall x[\alpha(x) \leftrightarrow \beta(x)] \rightarrow \varepsilon_{x} \alpha=\varepsilon_{x} \beta .
$$

From these WESP is easily derived, and so, a fortiori, SLEM.

The second approach is to take the $\varepsilon$-axiom in the (classically equivalent) form

$$
\alpha\left(\varepsilon_{x} \alpha\right) \vee \forall x \neg \alpha(x) .
$$

From this we can intuitionistically derive SLEM as follows:

Given a sentence $\beta$, define $\alpha(x)$ to be the formula

$$
(x=0 \wedge \beta) \vee(x=1 \wedge \neg \beta) .
$$

Then from (*) we get 


$$
\left[( \varepsilon _ { x } \alpha = 0 \wedge \beta ) \vee \left(\left[\left(\varepsilon_{x} \alpha=1 \wedge \neg \beta\right)\right] \vee \forall x \neg[(x=0 \wedge \beta) \vee(x=1 \wedge \neg \beta)],\right.\right.
$$

which implies

$$
[\beta \vee \neg \beta) \vee[\forall x \neg(x=0 \wedge \beta) \wedge \forall x \neg(x=1 \wedge \neg \beta)]
$$

whence

$$
[\beta \vee \neg \beta) \vee[\neg \beta \wedge \neg \neg \beta]
$$

winding up with

$$
\beta \vee \neg \beta \text {. }
$$

The third method is to allow $\varepsilon$ to act on pairs of formulas, each with a single free variable. Here, for each pair of formulas $\alpha(x), \beta(x)$ we introduce the "relativized" $\varepsilon$-term $\varepsilon_{x} \alpha / \beta$ and the "relativized" $\varepsilon$-axioms

$$
\text { (1) } \exists x \beta(x) \rightarrow \beta\left(\varepsilon_{x} \alpha / \beta\right) \quad \text { (2) } \exists x[\alpha(x) \wedge \beta(x)] \rightarrow \alpha\left(\varepsilon_{x} \alpha / \beta\right) .
$$

That is, $\varepsilon_{x} \alpha / \beta$ may be thought of as an individual that satisfies $\beta$ if anything does, and which in addition satisfies $\alpha$ if anything satisfies both $\alpha$ and $\beta$. Notice that the usual $\varepsilon$-term $\varepsilon_{x} \alpha$ is then $\varepsilon_{x} \alpha / x=x$. In the classical $\varepsilon$-calculus $\varepsilon_{x} \alpha / \beta$ may be defined by taking

$$
\varepsilon_{x} \alpha / \beta=\varepsilon_{y}\left[\left[y=\varepsilon_{x}(\alpha \wedge \beta) \wedge \exists x(\alpha \wedge \beta)\right] \vee\left[y=\varepsilon_{x} \beta \wedge \neg \exists x(\alpha \wedge \beta)\right]\right] .
$$

But the relativized $\varepsilon$-scheme is not derivable in the intuitionistic $\varepsilon$-calculus since it can be shown to imply SLEM. To see this, given a formula $\gamma$ define

$$
\alpha(x) \equiv x=1 \quad \beta(x) \equiv x=0 \vee \gamma
$$

Write $a$ for $\varepsilon_{x} \alpha / \beta$. Then we certainly have $\exists x \beta(x)$, so (1) gives $\beta(a)$, i.e.

$$
a=0 \vee \gamma
$$

Also $\exists x(\alpha \wedge \beta) \leftrightarrow \gamma$, so (2) gives $\gamma \rightarrow \alpha(a)$, i.e.

$$
\gamma \rightarrow a=1
$$

whence

$$
a \neq 1 \rightarrow \neg \gamma,
$$


so that

$$
a=0 \rightarrow \neg \gamma
$$

And the conjunction of this with (3) gives $\gamma \vee \neg \gamma$, as claimed.

\section{References}

[1] Bell, John L. Hilbert's epsilon operator in intuitionistic type theories, Math. Logic Quarterly, 39, 1993.

[2] Bell, John L. Hilbert's epsilon-operator and classical logic, Journal of Philosophical Logic, 22, 1993.

[3] Grayson, R. J. Heyting-valued models for intuitionistic set theory. In Fourman, M. P., Mulvey, C. J., and Scott, D. S. (eds.) (1979) Applications of Sheaves. Proc. L.M.S. Durham Symposium 1977. Springer Lecture Notes in Mathematics 753, pp. 402-414.

[4] Lawvere, F.W. and Rosebrugh, R. Sets for Mathematics. Cambridge University Press, 2003. 